\title{
Actualización: Novedades en el reporte (PRISMA) y en la evaluación de la calidad (AMSTAR) de las revisiones sistemáticas y los meta-análisis
}

News in report (PRISMA) and evaluation of the quality (AMSTAR) of systematic reviews and meta-analysis Agustín Ciapponi*

\begin{abstract}
Resumen
Considerando los avances conceptuales y prácticos en la ciencia de las revisiones sistemáticas, en este artículo el autor resume dos listas de cotejo: 1) una de ellas (PRISMA) sintetiza el consenso para el reporte de ítems de revisiones sistemáticas y meta-análisis); 2) la otra (AMSTAR) es un Instrumento para evaluar la calidad metodológica de las revisiones sistemáticas.

\section{Abstract}

Considering practical and conceptual advances in the science of the systematic reviews, the author summarizes two lists of comparison: 1) one of them (PRISMA) synthesizes consensus about reporting ítems for systematic reviews and meta-analysis); 2) the other (AMSTAR) is an Instrument to evaluate the methodological quality of systematic reviews.
\end{abstract}

Palabras clave: revisiones sistemáticas, meta-análisis, calidad metodológica. Key words: systematic reviews, meta-analysis, metodologic quality.

Ciapponi A, Novedades en el reporte (PRISMA) y la evaluación de la calidad (AMSTAR) de las revisiones sistemáticas y los meta-análisis. Evid Act Pract Ambul. 12(4): 141-145-Oct-Dic.2009.

\section{Introducción}

Las revisiones sistemáticas (RS) y los meta-análisis (MA) son herramientas esenciales para resumir la evidencia con precisión y confiabilidad. Ayudan a los profesionales a mantenerse actualizados; aportan evidencias a los encargados de formular políticas de salud para juzgar los riesgos y beneficios de las intervenciones sanitarias; reúnen y resumen la investigación relacionada con los pacientes; brindan un punto de partida para los desarrolladores de guías de práctica clínica; resumen las investigaciones previas para los financiadores que deseen apoyar nuevas investigaciones'; y ayudan a los editores a juzgar los méritos de publicación de nuevos estudios ${ }^{2}$. Datos recientes sugieren que al menos 2.500 nuevas revisiones sistemáticas en inglés se indexan anualmente en MEDLINE ${ }^{3}$, siendo considerable el incremento anual en las últimas dos décadas. Ver figuras 1.

Figura 1. Incremento anual de RS y MA PubMed: "MetaAnalysis"[MeSH] OR "systematic review"[tw]= 17.059

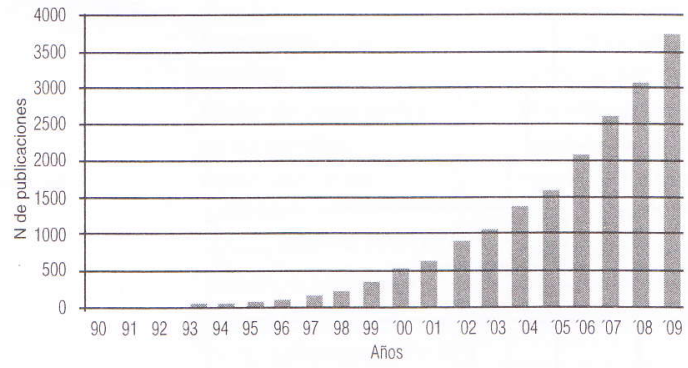

Desgraciadamente, existe considerable evidencia de que en las RS la información clave es a menudo mal reportada, lo que disminuye su potencial utilidad ${ }^{3 \cdot 6}$. Como ocurre con todas las actividades de investigación, las RS deben ser plena y transparentemente reportadas para permitir a los lectores evaluar las fortalezas y debilidades de la investigación ${ }^{7}$.

En 1996 y para hacer frente a la subóptima presentación de reportes de los MA, un grupo internacional desarrolló una guía denominada declaración QUOROM (QUality Of Reporting Of Meta-analyses: Calidad de Reporte de Meta-análisis) centrada en el reportes de MA de ensayos controlados aleatorizados ${ }^{8}$. Considerando los avances conceptuales y prácticos en la cien- cia de las RS (ver Recuadro 1), se resume una revisión actualizada de estas guía, denominada PRISMA (Preferred Reporting Items for Systematic reviews and Meta-Analyses: Reporte preferido de ĺtems para Revisiones Sistemáticas y MetaAnálisis $)^{9,10}$

Si bien la lista de cotejo PRISMA también puede ser útil para la evaluación crítica de las RS publicadas, no es un instrumento de evaluación de la calidad de una RS. Para remarcar las diferencias entre el reporte y la evaluación de la calidad, y para completar la actualización en este campo se presentará también el más reciente instrumento de evaluación de la calidad de RS y MA denominado AMSTAR (A MeaSurement Tool to Assess the methodological quality of systematic Reviews: Un Instrumento de Medición para Evaluar la calidad metodológica de las Revisiones sistemáticas) $)^{1+113}$

\section{Sobre la terminología utilizada}

La terminología utilizada para describir las RS y MA ha evolucionado con el tiempo y varía según el campo. Quizás esto explica que diferentes términos han sido utilizados por diferentes grupos (educadores y psicólogos).

Recordamos que la realización de una RS se compone de varios pasos explícitos y reproducibles, tales como la identificación de todos los posibles registros de estudios, la selección de los estudios elegibles, la evaluación de la probabilidad de sesgo, la extracción de datos, la síntesis cualitativa de los estudios incluidos, y, posiblemente, el MA.

Al principio todo este proceso se denominó MA y fue definido en la declaración QUOROM ${ }^{8}$. Más recientemente y en especial en el campo de la investigación en salud, ha habido una tendencia a preferir el término RS; reservando el término MA sólo para la etapa en la que se realiza la síntesis cuantitativa.

La Colaboración Cochrane utiliza esta terminología ${ }^{14}$, en virtud del cual un MA, si se realiza, es un componente de una RS.

Independientemente de la complejidad de la cuestión, siempre es posible completar una RS de los datos existentes, pero no siempre es posible, ni deseable, sintetizar cuantitativamente los resultados, debido a diferencias clínicas, metodológicas o estadísticas entre los estudios incluidos. 
Por el contrario, con la acumulación prospectiva de posibles estudios y datos en donde el plan es eventualmente combinarlos, el término "MA prospectivo", puede tener más sentido que el de "RS".

Para esfuerzos retrospectivos, una posibilidad es dar el término RS a todo el proceso, hasta el momento cuando se decide si debe llevarse a cabo una síntesis cuantitativa.

Si ésta se lleva a cabo, algunos investigadores se refieren a la misma como un MA. Esta definición es similar a la que se encuentra en la actual edición del Diccionario de Epidemiología $^{15}$.

Si bien reconocemos que el uso de estos términos es inconsis tente y hay un desacuerdo residual entre los miembros del grupo de trabajo PRISMA, hemos adoptado las definiciones utilizadas por la Colaboración Cochrane ${ }^{14}$.

El cuadro 1 resume el significado estipulado de la terminología utilizada en estos reportes.
Cuadro 1: significado estipulado de la terminología utilizada en estos reportes.

\section{Revisión sistemática}

Intenta reunir todas las pruebas empíricas que se ajusten a los criterios de elegibilidad pre-especificados para responder a una pregunta de investigación. Utiliza explícita y sistemáticamente métodos seleccionados con miras a reducir los sesgos al mínimo, lo que da resultados confiables a partir de la cual se pueden sacar conclusiones y adoptar decisiones ${ }^{16,17}$. Las principales características de una RS son los siguientes:

a) Claro conjunto de objetivos, con una metodología explícita y reproducible.

b) Búsqueda sistemática que trate de identificar todos los estudios que se ajusten a los criterios de elegibilidad.

c) Evaluación de la validez de los hallazgos de los estudios incluidos (ej. probabilidad de sesgo).

d) Presentación sistemática y síntesis de las características y de los hallazgos de los estudios incluidos.

Meta-análisis

Uso de técnicas estadísticas para integrar y sintetizar los resultados de los estudios incluidos. Al combinar la información de todos los estudios pertinentes, los MA pueden proporcionar estimaciones más precisas de los efectos de la atención de la salud que las derivadas de los estudios individuales incluidos en una RS.

Figura 3: flujo de información a través de las diferentes fases de una revisión sistemática.

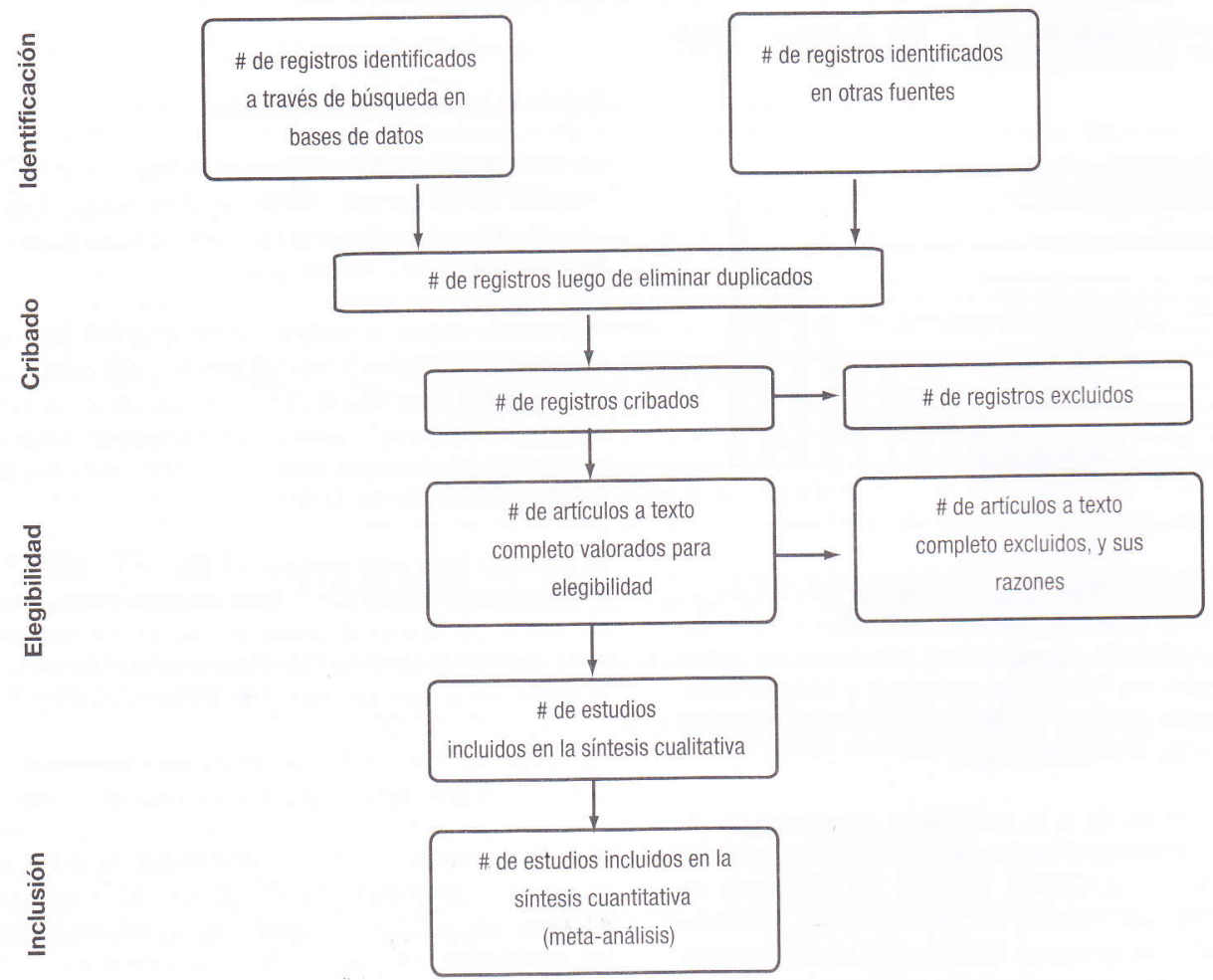


abla 1. Lista de cotejo de los puntos a incluir al reportar una revisión sistemática o un meta-análisis.

\begin{tabular}{|c|c|c|c|}
\hline - Sección & Tópico & $\#$ & Reportado en página \\
\hline Título & & 1 & Identificar el reporte como una revisión sistemática, un meta-análisis o ambos. \\
\hline Resumen estructurado & & 2 & $\begin{array}{l}\text { Proporcionar un resumen estructurado incluyendo, si es aplicable: antecedentes (background); objetivos; fuentes de } \\
\text { datos; criterios de elegibilidad del estudio, participantes e intervenciones; métodos de la valoración y de la síntesis } \\
\text { del estudio; resultados; limitaciones; conclusiones e implicancias de los resultados principales; número de registro } \\
\text { de la revisión sistemática. }\end{array}$ \\
\hline \multirow[t]{2}{*}{ Introducción } & Justificación & 3 & Describir la justificación para la revisión en el contexto ya conocido. \\
\hline & Objetivos & 4 & $\begin{array}{l}\text { Explicitar las preguntas planteadas referentes a participantes, intervenciones, comparaciones, resultados y al diseño } \\
\text { del estudio (PICOS del inglés). }\end{array}$ \\
\hline \multirow{12}{*}{ Métodos } & Protocolo y del registro & 5 & $\begin{array}{l}\text { Indicar si existe un protocolo de la revisión, y en ese caso cómo puede ser consultado. (ej. dirección Web), y, si está } \\
\text { disponible, proporcionar la información del registro incluyendo el número de registro. }\end{array}$ \\
\hline & $\begin{array}{l}\text { Criterios de la } \\
\text { elegibilidad }\end{array}$ & 6 & $\begin{array}{l}\text { Especificar las características del estudio (ej. PICOS, longitud del seguimiento) y reporte las características (ej. años } \\
\text { considerados, lengua, estado de la publicación) usadas como criterios para la elegibilidad, justificándolo. }\end{array}$ \\
\hline & $\begin{array}{l}\text { Fuentes de } \\
\text { información }\end{array}$ & 7 & $\begin{array}{l}\text { Describir todas las fuentes de información de la búsqueda (ej. bases de datos con las fechas de cobertura, contacto } \\
\text { con los autores del estudio para identificar estudios adicionales) y la fecha de última búsqueda. }\end{array}$ \\
\hline & Búsqueda & 8 & $\begin{array}{l}\text { Presentar una descripción completa de al menos una estrategia de búsqueda electrónica, incluyendo cualquier límite } \\
\text { usado, de forma tal que pueda ser repetida. }\end{array}$ \\
\hline & $\begin{array}{l}\text { Selección de } \\
\text { estudios }\end{array}$ & 9 & $\begin{array}{l}\text { Indicar el proceso de selección de los estudios (cribado, elegibilidad, inclusión en la revisión sistemática, y, si fuera } \\
\text { aplicable, en el meta-análisis). }\end{array}$ \\
\hline & $\begin{array}{l}\text { Proceso de recolección } \\
\text { de datos }\end{array}$ & 10 & $\begin{array}{l}\text { Describir el método de extracción de datos de los reportes (ej. formularios pilotados, independientemente, en } \\
\text { duplicado) y de cualquier proceso para obtener y confirmar datos de los investigadores. }\end{array}$ \\
\hline & $\begin{array}{l}\text { İtems de } \\
\text { datos }\end{array}$ & 11 & $\begin{array}{l}\text { Enumerar y definir todas las variables para las cuales los datos fueron buscados (ej. PICOS, fuentes de financiación) } \\
\text { y cualquier asunción o simplificación hecha. }\end{array}$ \\
\hline & $\begin{array}{l}\text { Riesgo de sesgo } \\
\text { en los estudios } \\
\text { individuales }\end{array}$ & 12 & $\begin{array}{l}\text { Riesgo de sesgo en los estudios individuales. Describir los métodos usados para determinar el riesgo de sesgo en } \\
\text { los estudios individuales (especificando si fue hecho a nivel de estudio, de resultado o de ambos) y cómo esta } \\
\text { información es utilizada en la síntesis de los datos. }\end{array}$ \\
\hline & Medidas sumarias & 13 & Indicar las medidas sumarias principales (ej. riesgo relativo, diferencia de medias). \\
\hline & $\begin{array}{l}\text { Sintesis de } \\
\text { resultados }\end{array}$ & 14 & $\begin{array}{l}\text { Describir los métodos para manejar datos y combinar resultados de los estudios, si está hecho, incluyendo medidas } \\
\text { de consistencia }(\mathrm{Ej}, \text { I2) para cada meta-análisis. }\end{array}$ \\
\hline & $\begin{array}{l}\text { Riesgo de sesgo a } \\
\text { través de los estudios }\end{array}$ & 15 & $\begin{array}{l}\text { Especificar cualquier valoración del riesgo de sesgo que pueda afectar a la evidencia acumulada (ej. sesgo de } \\
\text { publicación, reporte selectivo dentro de estudios). }\end{array}$ \\
\hline & $\begin{array}{l}\text { Análisis } \\
\text { adicionales }\end{array}$ & 16 & $\begin{array}{l}\text { Describir los métodos de análisis adicionales (ej. análisis de la sensibilidad o de subgrupo, meta-regresión), si están } \\
\text { hechos, indicando cuáles fueron pre-especificados. }\end{array}$ \\
\hline \multirow{7}{*}{ Resultados } & $\begin{array}{l}\text { Selección de } \\
\text { estudios }\end{array}$ & 17 & $\begin{array}{l}\text { Dar el número de estudios cribados, valorados para elegibilidad, e incluidos en la revisión, con las razones de } \\
\text { exclusión en cada etapa, idealmente con un diagrama de flujo. }\end{array}$ \\
\hline & $\begin{array}{l}\text { Características de los } \\
\text { estudios }\end{array}$ & 18 & $\begin{array}{l}\text { Para cada estudio, presentar las características para las cuales fueron extraidos los datos (ej. el tamaño del estudio, } \\
\text { PICOS, período de seguimiento) y proporcionar las citas. }\end{array}$ \\
\hline & $\begin{array}{l}\text { Riesgo de sesgo dentro } \\
\text { de los estudios }\end{array}$ & 19 & $\begin{array}{l}\text { Presentar los datos sobre el riesgo de sesgo de cada estudio y, si está disponible, dar cualquier valoración a nivel de } \\
\text { resultado (ver el punto 12). }\end{array}$ \\
\hline & $\begin{array}{l}\text { Resultados de los } \\
\text { estudios } \\
\text { individuales }\end{array}$ & 20 & $\begin{array}{l}\text { Para todos los resultados considerados (beneficios y daños), presentar, para cada estudio: (a) los datos sumarios } \\
\text { simples para cada grupo de intervención y (b) las estimaciones del efecto y los intervalos de confianza, idealmente } \\
\text { con un diagrama (forest plot). }\end{array}$ \\
\hline & Síntesis de resultados & 21 & Presentar los resultados de cada meta-análisis hecho, incluyendo intervalos de confianza y medidas de consistencia. \\
\hline & $\begin{array}{l}\text { Riesgo de sesgo a } \\
\text { través die los estudios }\end{array}$ & 22 & Presentar los resultados de cualquier valoración de riesgo de sesgo a través delos estudios (ver punto 15). \\
\hline & $\begin{array}{l}\text { Análisis } \\
\text { adicionales }\end{array}$ & 23 & $\begin{array}{l}\text { Dar los resultados de análisis adicionales, si estuvieran hechos (ej. análisis de la sensibilidad o de subgrupo, meta- } \\
\text { regresión [ver el punto 16]) }\end{array}$ \\
\hline \multirow[t]{3}{*}{ Discusión } & $\begin{array}{l}\text { Resumen de la } \\
\text { evidencia }\end{array}$ & 24 & $\begin{array}{l}\text { Resumir los resultados principales incluyendo la fuerza de la evidencia de cada resultado principal; considerar su } \\
\text { importancia para los principales grupos (ej. proveedores de salud, usuarios, y decisores). }\end{array}$ \\
\hline & Limitaciones & 25 & $\begin{array}{l}\text { Discutir las limitaciones a nivel de estudio y de resultado (ej. riesgo de sesgo) y a nivel de la revisión (ej. } \\
\text { recuperación incompleta de la evidencia identificada, reportando sesgos). }\end{array}$ \\
\hline & Conclusiones & 26 & $\begin{array}{l}\text { Proporcionar una interpretación general de los resultados en el contexto de otra evidencia, y las implicancias para } \\
\text { futuras investigaciones. }\end{array}$ \\
\hline \multicolumn{2}{|l|}{ Financiamiento } & 27 & $\begin{array}{l}\text { Describir las fuentes de financiamiento para la revisión 'sistemática y otras ayudas (ej. fuente de datos); rol de los } \\
\text { financiadores para la revisión sistemática. }\end{array}$ \\
\hline
\end{tabular}

Reporte de revisiones sistemática y meta-análisis: la lista de cotejo PRISMA

La lista de cotejo PRISMA consta de 27 puntos o ítems (ver tabla 1) y de un diagrama de flujo de cuatro fases (ver figura 3) especialmente diseñado para evaluar el reporte de RS y MA de ensayos clínicos aleatorizados.
La mayor parte de los ítems son pertinentes para reportar $\mathrm{RS}$ de estudios no aleatorizados sobre beneficios y daños de las intervenciones; pero si se abordan cuestiones relativas a la etiología, diagnóstico, pronóstico o revisiones epidemiológicas pueden necesitarse modificaciones o incorporar elementos adicionales. La lista PRISMA debería sustituir al QUOROM en las revistas que lo habían avalado y es de esperar que otras revistas también lo 
hagan. Además de la declaración PRISMA, se produjo un documento de apoyo de explicación y elaboración ${ }^{10}$. que incluye una amplia base de datos de ejemplos para poner de relieve la mejor manera de reportar cada punto de la lista y la identificación de una amplia base de evidencia para apoyar la inclusión de cada punto. Al igual que cualquier esfuerzo basado en la evidencia, PRISMA es un documento vivo.

Con este fin, los lectores están invitados a hacer comentarios sobre la versión revisada, en particular la nueva lista de cotejo PRISMA y el diagrama de flujo, a través del sitio Web PRISMA (http://www.prisma-statement.org/) para el continuo desarrollo de PRISMA
Evaluación de la calidad metodológica de las revisiones sistemáticas: el instrumento AMSTAR

AMSTAR (A MeaSurement Tool to Assess the methodologica quality of systematic Reviews) es un instrumento de medición para evaluar la calidad metodológica de las revisiones sistemáticas que consta de 11 ítems (ver tabla 2) desarrollados por un reconocido grupo de expertos en estrecha vinculación con la Colaboración Cochrane y el grupo PRISMA. La herramienta consta de 11 ítems y tiene buena validez de constructo* y contenido* y confiabilidad* para evaluar la calidad metodológica de las revisiones sistemáticas. Aun cuando este instrumento ha sido fehacientemente probado ${ }^{12+3}$, sería conveniente que sea consolidado y eventualmente refinado mediante una más amplia gama de evaluadores y de revisiones.

Tabla 2. Instrumento de evaluación de la calidad de revisiones sistemáticas y meta-análisis.

\begin{tabular}{|c|c|c|c|c|}
\hline & SI & No & SR & NA \\
\hline $\begin{array}{l}\text { 1. ¿Se brindó un diseño "a priori"? } \\
\text { La pregunta de investigación y los criterios inclusión deben ser definidos antes de realizar la revisión. }\end{array}$ & & & & \\
\hline $\begin{array}{l}\text { 2. ¿Hubo selección de estudios y extracción de datos por duplicado? } \\
\text { Debe haber al menos dos extractores de datos independientes y un procedimiento de consenso establecido para los } \\
\text { desacuerdos. }\end{array}$ & & & & \\
\hline $\begin{array}{l}\text { 3. ¿Se llevó a cabo una búsqueda exhaustiva de la literatura? } \\
\text { Debe buscarse en al menos dos fuentes electrónicas. El reporte debe incluir los años y las bases de datos utilizadas } \\
\text { (Ejemplo, Central, EMBASE y MEDLINE). Deben mencionarse las palabras clave y/o los términos MESH y si fuera } \\
\text { factible, debería brindarse la estrategia de búsqueda. Todas las búsquedas deben ser complementadas consultando } \\
\text { current contents*, revisiones, libros de texto, registros especializados o a expertos en el campo de estudio en } \\
\text { particular, y examinando las referencias en los estudios encontrados. }\end{array}$ & & & & \\
\hline $\begin{array}{l}\text { 4. ¿Se usó el estado de publicación (ej. literatura gris) como un criterio de inclusión? } \\
\text { Los autores deben afirmar que buscaron reportes sin considerar su tipo de publicación. Los autores deben afirmar si } \\
\text { excluyeron o no cualquier reporte (de la revisión sistemática), basados en su estado de publicación, idioma, etc. }\end{array}$ & & & & \\
\hline $\begin{array}{l}\text { 5. ¿Se proporcionó una lista de estudios (incluidos y excluidos)? } \\
\text { Debe suministrarse una lista de los estudios incluidos y excluidos. }\end{array}$ & & & & \\
\hline $\begin{array}{l}\text { 6. ¿Fueron suministradas las características de los estudios incluidos? } \\
\text { Deben suministrarse los datos de los estudios originales, de forma agregada como en una tabla, sobre los } \\
\text { participantes, las intervenciones y los resultados. Deben ser reportados los rangos de las características en todos los } \\
\text { estudios analizados por ejemplo, edad, raza, sexo, datos socioeconómicos relevantes, estado de enfermedad, } \\
\text { duración, gravedad, u otras enfermedades. }\end{array}$ & & & & \\
\hline $\begin{array}{l}\text { 7. ¿Fue valorada y documentada la calidad científica de los estudios incluidos? } \\
\text { Deben suministrarse los métodos de la valoración "a priori" (Ej, para estudios de efectividad, si el autor decidió } \\
\text { incluir solamente ensayos aleatorizados, doble-ciego y controlados con placebo, o la asignación oculta como criterio } \\
\text { de inclusión); para otros tipos de estudios, serán relevantes ítems alternativos. }\end{array}$ & & & & \\
\hline $\begin{array}{l}\text { 8. ¿Fue usada apropiadamente la calidad científica de los estudios incluidos para formular las } \\
\text { conclusiones? } \\
\text { Los resultados de rigor metodológico y la calidad científica deben ser considerados en el análisis y las conclusiones } \\
\text { de la revisión, y declarado explícitamente para formular las recomendaciones. }\end{array}$ & & & & \\
\hline $\begin{array}{l}\text { 9. ¿Fueron apropiados los métodos utilizados para combinar los resultados de los estudios? } \\
\text { Para los resultados combinados, debe realizarse una prueba para asegurar que los estudios eran combinables, que } \\
\text { valore su homogeneidad (Ej, la prueba de Chi2 para la homogeneidad, }{ }^{2} \text { ). Si existiese heterogeneidad debe usarse } \\
\text { un modelo de efectos aleatorios y/o considerar si es clínicamente apropiado la combinación (Ej, ¿Es sensato } \\
\text { combinar?). }\end{array}$ & & & & \\
\hline $\begin{array}{l}\text { 10. ¿Fue valorada la probabilidad de sesgo de publicación? } \\
\text { Una valoración del sesgo de publicación debe incluir una combinación de las ayudas gráficas (Ej, gráfico de embudo } \\
\text { u otras pruebas disponibles) y/o pruebas estadísticas (Ej, prueba de regresión de Egger). }\end{array}$ & & & & \\
\hline $\begin{array}{l}\text { 11. ¿Fue incluido el conflicto de intereses? } \\
\text { Las fuentes potenciales de financiamiento deben ser claramente agradecidas tanto la revisión sistemática como los } \\
\text { estudios incluidos. }\end{array}$ & & & & \\
\hline
\end{tabular}

SR: sin respuesta. NA: no aplicable. 
Referencias

1.Canadian Institutes of Health Research Randomized controlled trials registration/application c. Available: http://www.cihr-irsc.gc.ca/e/documents/rct_reg_e.pdf://www.cihr-irsc.gc.ca/e/documents/rct reg_e.pdf. Accessed 26 May 2009.

2. Young C. Horton RPctic.107-8.

3. Moher D y col. Reporting characteristics of systematic reviews. PLoS Med 4: e78. doi:10.1371/journal.pmed:10.1371/journal.pmed.0040078.

4. Dixon E, Hameed M, Sutherland F, Cook DJ, Doig CEm-aitgs|Aca.450-9.

5. Hemels ME, Vicente C, Sadri H, Masson MJ, Einarson TRQaom-aoRopimdd.477-84.

6. Jin W y col. (2008) The reporting quality of meta-analyses improves: A random sampling study:770-5.

7. Moher D, Simera I, Schulz KF. Hoey J, Altman DGHe, peer $\mathrm{r}$, et al.:13.

8. Altman D y col. KEQUATORRgthr.1149-50

9. Moher D y col.Preferred reporting items for systematic reviews and meta-analyses: the PRISMA statement. PLoS Med 2009;6(7):e1000097.

10. Liberati Ay col. The PRISMA statement for reporting systematic reviews and meta-analyses of studies that evaluate health care interventions: explanation and elaboration. PLoS Med 2009:6(7):e1000100.

11. Shea BJ, Hamel C, Wells GA, Bouter LM, Kristjansson E, Grimshaw J, et al. AMSTAR is a reliable and valid measurement tool to assess the methodological quality of systematic reviews. J Clin Epidemiol 2009.

12. Shea B y col. External validation of a measurement tool to assess systematic reviews (AMSTAR). PLoS One 2007;2(12):e1350.

13. Shea B y col.Development of AMSTAR: a measurement tool to assess the methodological quality of systematic reviews. BMC Med Res Methodol 2007:7:10.

14. Green S y col. (2008) Chapter 1: What is a systematic review? In: Higgins JPT, Green S, editors. Cochrane handbook for systematic reviews of interventions version 5.0 .0 [updated

February 2008]. The Cochrane Collaboration. Available: http://www.cochrane-handbook.org://www.cochrane-handbook.org/. Accessed 26 May 2009.

15. Last JMAdoe.Oxford University Press \& International Epidemiological Association.

16. Antman $\mathrm{E}$ y col. CAcorom-aorct, recommendations of clinical e. Treatments for myocardial infarction:240-8.

17. Oxman A y col. HTsorr. Ann N Y Acad Sci 703: 125-133:125-33. discussion 33-4.

\section{H \\ HOSPITAL ITALIANO}

de Buenos Aires

Inicio de Actividades:

15 de Abril de 2010

Duración: Dos años

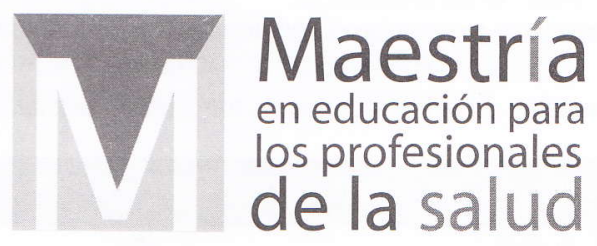

Resolución Ministerial 1074/2009

Director: Prof. Dr. Eduardo Durante

Co-Director: Prof. Dr. Carlos Brailovsky

\section{Apertura de Inscripción Octubre de 2009}

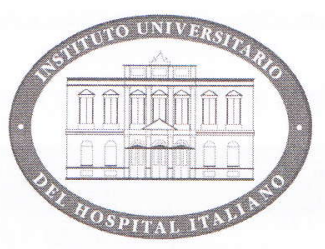

MODALIDAD

SEMIPRESENCIAL

Destinatarios: Profesionales de la Salud (Médicos, Odontólogos, Lic. en Enfermería, en Psicología,

en Psicopedagogía, en Nutrición, en Fonoaudiología, en Terapia Ocupacional, kinesiólogos, Bioquímicos,

Farmacéuticos) u otros profesionales egresados de universidades nacionales o

extranjeras con título equivalente.

\section{Objetivos}

- Capacitar a los profesionales de la salud para diseñar y evaluar programas educacionales.

- Reflexionar sobre su práctica y generar alternativas fundamentadas.

Informes E Inscripción:

- Enseñar y promover el aprendizaje activo entre los alumnos.

Instituto Universitario Escuela de Medicina Hospital Italiano

- Participar de experiencias educativas innovadoras.

Potosí 4249 Oficina Posgrado

- Comprender, interpretar y diseñar trabajos científicos en el área educativa.

TEL. 4959-0200. Int. 5324

- Desarrollar habilidades de gestión y liderazgo.

Horario: De lunes a viernes de 10.00 a
19.00 horas

posgrado@hospitalitaliano.org.ar 